\title{
Impacting and Wetting Dynamics of Single Polysilazane Droplet on Inconel Surface
}

\author{
Fujun Wang, Tosin Ajayi, Kaushik Nonavinakere Vinod, Chengying Xu, Tiegang Fang* \\ Department of Mechanical and Aerospace Engineering, North Carolina State University, \\ Raleigh, North Carolina, 27695, United States \\ *Corresponding author email: tfang2@ncsu.edu
}

\begin{abstract}
Silicon-based polymers are promising materials for making polymer-derived ceramic (PDC) coatings for a variety of applications. One way to obtain thermally stable and chemically resistant PDCs is to, firstly, spray liquid polymers onto the target surface and then, cure these precursors through thermal, radiative, or chemical approach. Yet, the interactions during the spray coating process between those polymer droplets and target surfaces are not well characterized throughout current literature. This study presents a detailed characterization of the single droplet impacting and spreading dynamics for a specific type of ceramic precursor, Polysilazane, on the Inconel surface, which has wide applications in seawater and aerospace fields. In the experiment, the Polysilazane droplet diameter was fixed around $1.6 \mathrm{~mm}$ while its impact velocity was varied between $0.2 \mathrm{~m} / \mathrm{s}$ and $4.2 \mathrm{~m} / \mathrm{s}$. The same parameter space was repeated with water droplets for comparison. High-speed $(4,400 \mathrm{fps})$, short $(<2 \mathrm{~s})$ videos and low-speed (30fps), long (>300s) videos were taken simultaneously to cover both the instantaneous impacting process and the whole slow spreading process. It is revealed that Polysilazane droplets have significantly different spreading characteristics from water droplets on Inconel surface. Water droplets show strong retraction after impacting on Inconel surface. However, for Polysilazane droplets, no retraction occurs. Instead, a slower wetting process continues after the "plateau" relaxation stage. Analysis of the droplet maximum spread factor shows that the variation of both liquids can be well correlated by an existing universal rescaling method.
\end{abstract}

\section{Keywords}

polymer droplet, droplet impact, wetting dynamics, ceramic coating

\section{Introduction}

A promising alternative approach for the fabrication of ceramic coatings is the polymer-derived ceramic (PDC) technology [1-3], which has been applied onto different substrate materials, such as carbon structures, $\mathrm{C} / \mathrm{SiC}$ composites, etc. The substrate material in this study is Inconel and it is one kind of typical materials used in gas turbine engines [4]. In the PDC processing, silicone-based polymers are first deposited on the substrate through spray coating [5]. Then, the conversion from the liquid polymer to solid ceramics takes place via thermal, chemical, or radiative heat treatment pro-cesses. These liquid polymers are often called preceramic polymer precursors, which usually have a backbone containing -Si-C(polycarbosilanes), -Si-N- (polysilazanes), or -Si-O- (polysiloxanes).

The thickness of the deposited precursor on the surface proves to be an important factor for the quality of the final coatings. A good control of the spray droplets and a fundamental understanding of their deposition process are necessary in order to have a suitable precursor layer for the subsequent curing process.

The impacting and spreading of a single droplet on a solid surface are usually regarded as the fundamental process for the more complicated interactions between the sprays and the 
surface. Briefly speaking, the post-impacting process can be classified into different stages: inertial, spreading, relaxation, wetting, and the final equilibrium [6]. Extensive research has been done in this area for simple liquids like water, which is well summarized in a few comprehensive reviews [7-8]. The inertial regime features a square root power law for the correlation of the spreading factor $\left(\beta=D(t) / D_{0}\right)$ as $\beta \sim \tau^{1 / 2}$, where $\tau=V_{0} t / D_{0}$ is the dimensionless time normalized by the initial droplet diameter $\left(D_{0}\right)$ and impact velocity $\left(V_{0}\right)$. In the later spreading stage, the evolution of the spreading factor depends on many different factors including the liquid properties, surface properties and the surrounding conditions. The maximum spread factor $\left(\beta_{m}\right)$, defined at the end of the spreading process, has been investigated a lot and significant advancements have been achieved in recent works [9-11]. In the succeeding relaxation phase, different outcomes can be observed for droplets on partially wettable surface, such as retraction [12], oscillation [13], partial rebound and total rebound. While for a complete wetting system, the relaxation stage usually appears as a plateau region [6] followed with a pure wetting stage, which brings the droplet to the final equilibrium. Such pure wetting process has been characterized by the Tanner's law [14]: $D(t) \sim t^{1 / 10}$ or $D(t) \sim t^{1 / 7}$.

Recently, complex liquid droplets, such as droplets of polymer solutions, have been gaining more focus because of their popularity in many industrial applications. Polymer additives into the aqueous phase has been known to prohibit the droplet rebound [15]. But the reason accounting for such phenomenon is not clear yet. The influence of the molecular weight of the polymer additive and the polymer concentration in the droplet post-impact process have also been investigated [16]. A higher averaged polymer molar mass will generally lead to a lower maximum spreading factor. The effect of the polymer concentration is more considerable during the retraction process. Moreover, the polymer concentration will have to surpass a certain threshold in order to alter the pure solvent droplet impact dynamics [16].

Most of the research above only studies the droplet post-impact process up to the relaxation phase (i.e., retraction and oscillation). A few other studies [17-18] successfully extended the time scale across several orders of magnitude to reveal the spreading characteristics in the wetting phase. It is shown in Ref. [18] that during the wetting phase, the complexed polymer solution droplet roughly exhibits Tanner's law for different impact velocities.

The reviewed work on the polymer-related droplet impact dynamics mostly deals with the diluted polymer solutions with a relatively low concentration. Whether those conclusions for simple liquid droplets remain valid for pure polymer liquid droplet is still questionable. This paper reports experimental measurements of the dynamic behaviours of pure Polysilazane droplets impacting on a specific type of Inconel surface with varying impact velocities. Extended time-scale recording serves together with the high-speed video photography to provide a whole history of the evolution of the pure polymer droplet impacting, spreading, and wetting process.

\section{Material and Methods}

The liquids used in the experiment include both the deionized (DI) water and the pure preceramic polymer. The pre-ceramic polymer is Polysilazane Durazane ${ }^{\circledR} 1800$, which is a thermally curable coating and high temperature resin. It is a colourless liquid and shows strong adhesion on many substrates. Its viscosity was measured by a Brookfield DV-E viscometer with spindle SC4-18 at different shear rates, which presents Newtonian fluid properties within the test range. Its surface tension was measured by the pendant drop method [19] via a customized setup, which was calibrated by deionized (DI) water. The properties of the DI water 
and Polysilazane were summarized in Table 1 for comparison. Droplets were generated by pushing a syringe pump equipped with a stainless-steel needle. The generated water droplets had a diameter around $1.9 \mathrm{~mm}$. The Polysilazane droplets had a diameter of $1.6 \mathrm{~mm}$. The droplets were released from heights between 3 and $1600 \mathrm{~mm}$, which resulted in varying impact velocities between $0.02 \mathrm{~m} / \mathrm{s}$ and $4.5 \mathrm{~m} / \mathrm{s}$.

Table 1 - Properties of water and Polysilazane

\begin{tabular}{c|ccc}
\hline Liquid & $\begin{array}{c}\text { Density } \\
\left(\mathrm{kg} / \mathrm{m}^{3}\right)\end{array}$ & $\begin{array}{c}\text { Surface tension } \\
(\text { dynes } / \mathrm{cm})\end{array}$ & $\begin{array}{c}\text { Dynamic } \\
\text { viscosity }(\mathrm{cp})\end{array}$ \\
\hline Water & 1.0 & 72.8 & 1 \\
Polysilazane & 1.0 & 25.3 & 32
\end{tabular}

The Inconel surface used in the experiment was prepared by cutting ATI 718TM sheet to small, square pieces. The pieces of Inconel surface were then sanded by a 1000-grit sandpaper. The roughness of the surfaces was measured using a stylus profilometer (Mitutoyo Sj-310) to be $R a \approx 0.2 \mu m$, which was considered as a smooth surface. The wettabilities of the Inconel were characterized by the sessile drop method using the same setup as described in Ref. [11].

The instantaneous droplet impacting process was recorded using a high-speed imaging camera (4,400 frames per second) from the side view. On the other side, a DSLR camera was utilized to record the slow but long wetting process. The obtained high-speed and low-speed videos were subsequently processed via a customized image processing code on MATLAB and corresponding spreading and wetting characteristics were quantified.

\section{Results and Discussion}

The dynamic process of the Polysilazane droplet impacting on Inconel surface will be analyzed in detail based on the experimental data. Comparison will be made between the Polysilazane and DI water droplets. In the following sections, the morphologies of the impacting droplets will first be characterized. The impacting, spreading and wetting process will be analyzed by quantifying the evolution of some key parameters such as spreading factor.

\section{Comparison of the impacting morphologies between water and Polysilazane droplets}

The experiments were conducted at 11 different impact velocities. To get an overview of the difference in the impacting morphologies between the water and Polysilazane droplets, we here demonstrate three representative cases with low, medium and relatively high impact velocities, respectively.

The case with very low impact velocity $\left(V_{0}=0.05 \mathrm{~m} / \mathrm{s}\right.$ for water, $V_{0}=0.02 \mathrm{~m} / \mathrm{s}$ for Polysilazane) is demonstrated in Fig. 1. The morphology observed for water droplets impacting on the Inconel surface is similar to what was reported in a recent literature [20] discussing droplet impact dynamics at relatively low impact velocities $(<1 \mathrm{~m} / \mathrm{s})$. In the early stage of the droplet impacting, the dynamic contact angle appears very high $\left(>160^{\circ}\right)$. As discussed in Ref. [20], such "pseudo-superhydrophobicity" of the Inconel surface should be caused by the thin air film entrapped between the liquid droplet and solid surface. The transition to wetting, due to the rupture of the air film, occurs at $3.4 \mathrm{~ms}$, which features a rapid decrease of the contact angle. Meanwhile, the capillary wave was excited at the three-phase contact line (TPCL) and starts propagating from the bottom to the top of the droplet. The water droplet reaches its maximum spread at $8.9 \mathrm{~ms}$. Compared to the water droplet, the morphology of the impacting Polysilazane droplet on the Inconel surface appears smoother. Instead of a sharp decrease, the contact angle of Polysilazane droplet decreases slowly over a long period as the droplet 
spreads on the surface. Moreover, the bending of the Polysilazane droplet interface at the TPCL is not as prominent as that for water droplet. These differences indicate that the wetting starts much earlier for Polysilazane droplet impacting on Inconel and it could be at the time as soon as the droplet touches the surface. Last but not the least, no propagation of capillary wave was found on the Polysilazane droplet interface.

(a) Water

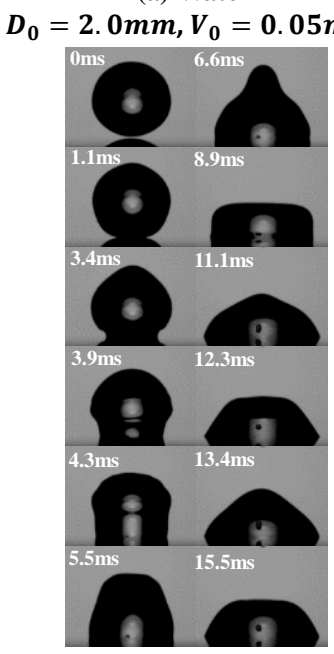

(b) Polysilazane

$D=1.6 \mathrm{~mm}, V=0.02 \mathrm{~m} / \mathrm{s}$

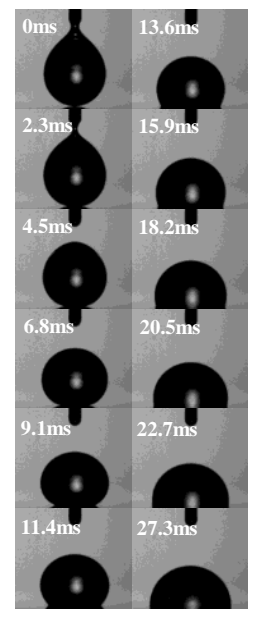

Figure 1. Low velocity impacting and spreading process for (a) water droplet and (b) Polysilazane droplet.

(a) Water $D=1.9 \mathrm{~mm}, V=1.3 \mathrm{~m} / \mathrm{s}$

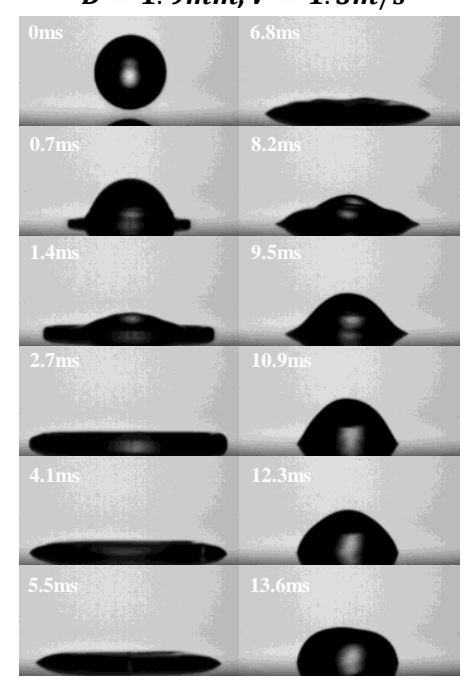

(b) Polysilazane

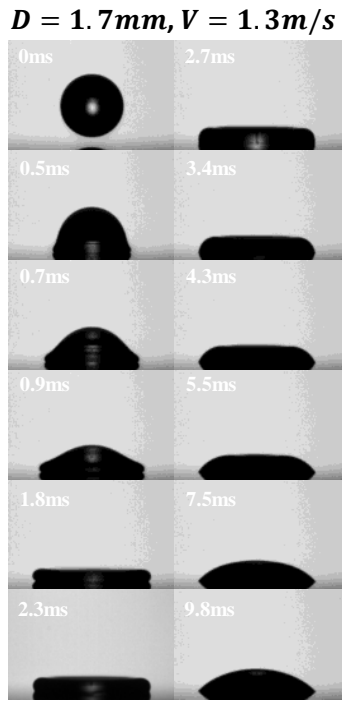

Figure 2. Medium velocity impacting and spreading process for (a) water droplet and (b) Polysilazane droplet.

When the impact velocity was raised above $1 \mathrm{~m} / \mathrm{s}$, the dominant effects of the air layer at low impact velocities disappeared for water droplets. Figures 2(a) and 2(b) demonstrate the impact process for water and Polysilazane droplets at $1.3 \mathrm{~m} / \mathrm{s}$. Water droplet retraction is dominant as shown in Fig. 2(a). The retraction process is the same as what was described in Ref. [12]. The water droplet reaches its maximum spread at $t=2.7 \mathrm{~ms}$, after which it starts retracting in the "inertial mode" until $t=5.5 \mathrm{~ms}$. In the later stage of the retraction, the "capillary mode" retraction dominates with an oscillation of the contact angle between $t=8.2 \mathrm{~ms}$ and $t=$ $12.3 \mathrm{~ms}$. In contrast, no retraction of the contact line was observed for Polysilazane droplet. The contact angle decreases from the large advancing contact angle $\left(>90^{\circ}\right)$ to a smaller one $\left(<90^{\circ}\right)$ but the TPCL remains pinned on the surface. This could be because of the large 
viscous dissipation or strong adhesion between the Polysilazane and Inconel surface. The detailed explanation of the pinning phenomena may be left for further investigation, which is beyond the scope of this paper. Another big difference that can be observed between the water and Polysilazane droplet is in their early stage of impacting process. The development of the lamella in impacting water droplet is obvious with a clear boundary dividing the upper truncated spherical droplet and the lower lamella part while it is not the case for Polysilazane droplet. The radial velocity of the impacting Polysilazane droplet is not that high compared to the impacting velocity. Therefore, the lamella will not go much beyond the bottom of the truncated sphere.

The morphologies of the droplet impacting with even higher velocity $(\sim 3.5 \mathrm{~m} / \mathrm{s})$ are summarized in Figs. 3(a) and 3(b). For the water droplet, its maximum spread $(t=3.0 \mathrm{~ms})$ nearly doubles as compared to the maximum spread $(t=2.7 \mathrm{~ms})$ in Fig. 2(a). However, for Polysilazane droplet, the increase in its maximum spread is much less. Finally, it should be noted that the last frames in Figs. 1(b), 2(b) and 3(b) do not necessarily represent the final equilibrium state of the Polysilazane droplet. The wetting of Polysilazane droplet on the Inconel surface was observed to continue even after the last image the high-speed camera can record. Therefore, the wetting process was also recorded by a DSLR camera from the side view.

(a) Water

$D=1.9 \mathrm{~mm}, V=3.5 \mathrm{~m} / \mathrm{s}$

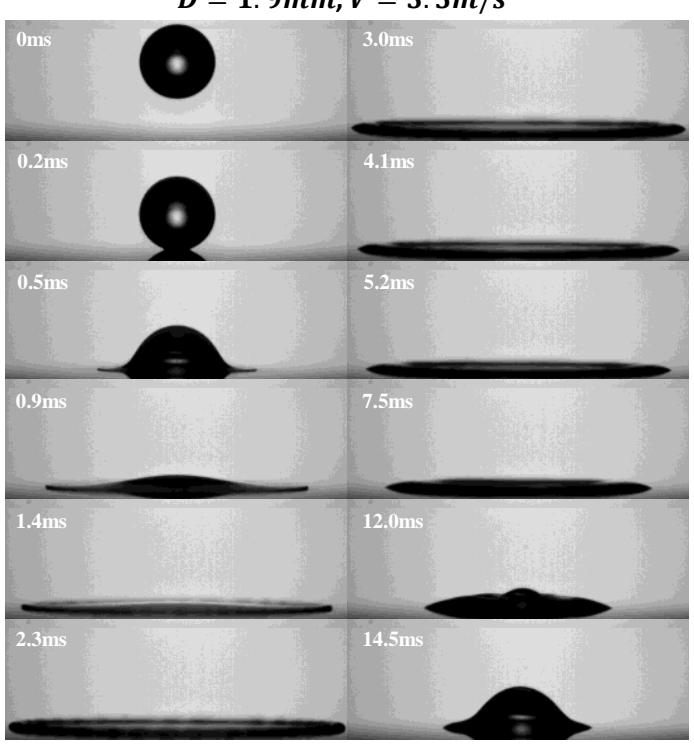

(b) Polysilazane

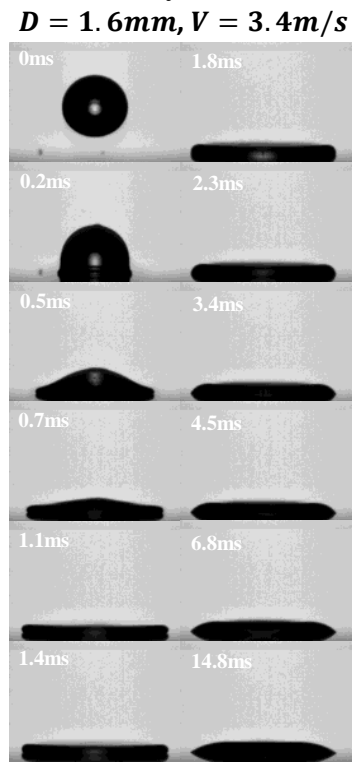

Figure 3. High velocity impacting and spreading process for (a) water droplet and (b) Polysilazane droplet.

\section{Quantification of the impacting, spreading and wetting process}

The time evolution of the spreading factor $(\beta)$ for water and Polysilazane droplets are demonstrated in Figs. 4(a) and 4(b). Normally, a droplet impacting onto a partially wettable surface can experience the following different phases [6]: kinematic, spreading, relaxation and equilibrium. The kinematic phase occurs at the very early stage of impact, in which the radial lamella is not visible. The evolution of the spreading factor, according to Ref. [6], should obey a universal power law. As shown in Fig. 4(c), a universal power law of $\beta=2.2 \tau^{0.5}$ can well describe the growth of the spreading factor in the kinematic phase $(\tau<0.1)$ for both water and Polysilazane droplets on the Inconel surface. After $\tau=0.1$, spreading curves diverge because factors other than the inertia kicks in, like the surface tension, surface wettability and liquid viscosity, etc. 
(a)

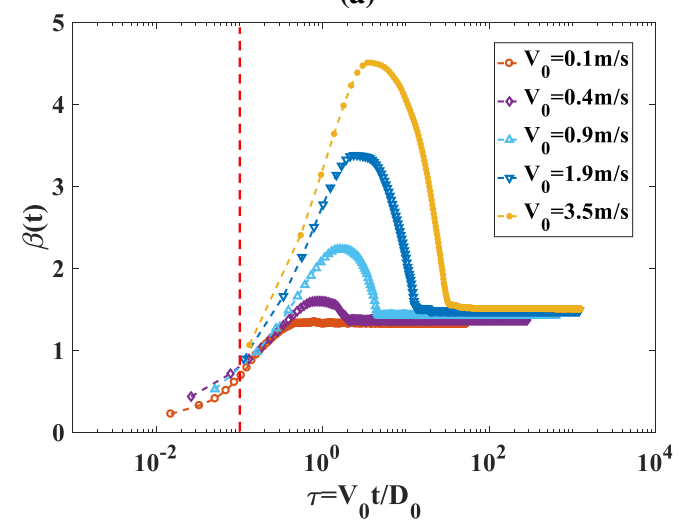

(c)

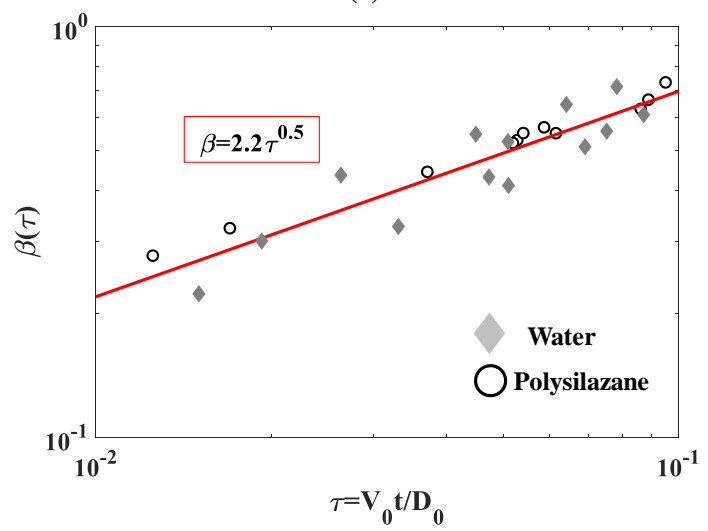

(b)

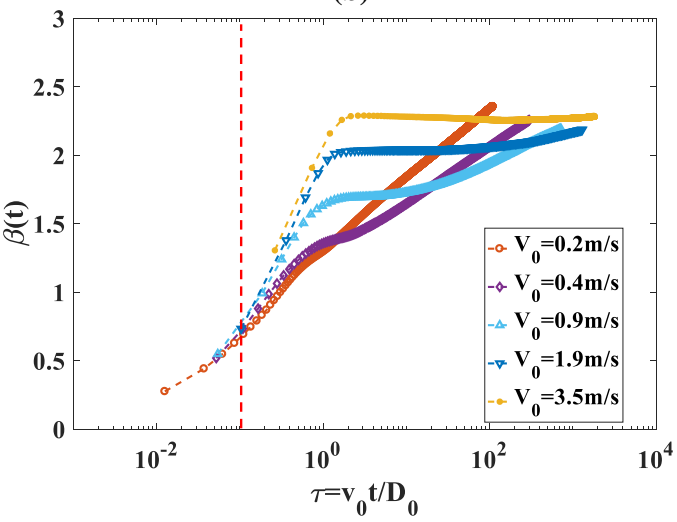

(d)

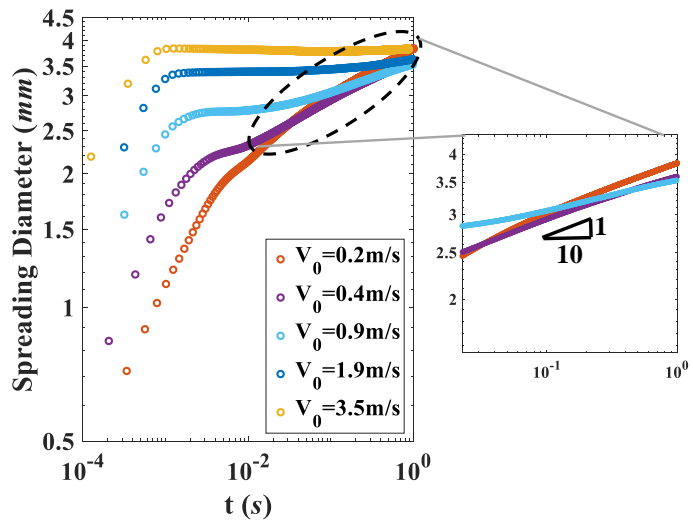

Figure 4. Time evolution of spreading factor for (a) water droplet and (b) Polysilazane droplet; (c) Evolution of spreading factor in kinematic phase; (d) Evolution of the dimensional spreading diameter with dimensional time for Polysilazane droplet.

For water droplet, the spreading curves will grow to the peak at the end of the spreading phase and then decrease and relax to the equilibrium position in the relaxation phase. The water droplet retraction becomes more dominant as the impact velocity increases. The characteristics of the water droplet retraction process appears similar to what was observed and described in a recent paper [12]. Soon after the retraction, the water droplet reaches its final equilibrium state, which is well defined by the flat spreading curves shown in Fig. 4(a). It is shown that the final equilibrium spreading factor $\left(\beta_{f}\right)$ is slightly bigger for droplet with a higher impact velocity.

For Polysilazane droplet, the spreading curves demonstrate distinct characteristics from the water droplets, especially in the relaxation phase. For these spreading curves, no "peak" can be defined as the spreading factor keeps growing even after $\tau=10^{2}$, which is beyond the recording length of the high-speed camera. This is typically the feature for droplet impacting and spreading on a completely wettable system, as reported in Ref. [6]. In this case, the "maximum spreading factor $\left(\beta_{m}\right)$ " corresponds to the maximum spreading only in the spreading phase, which is due to the inertial impacts. Therefore, the final spreading factor $\left(\beta_{f}\right)$, which incorporates the wetting effects, can be larger than $\beta_{m}$ for Polysilazane droplet impacting on Inconel surface. A transition phase exists between the inertial impacting phase and the wetting phase. In the case of high impact velocity, the transition phase features a long plateau stage. In the transition, the Polysilazane droplet relaxes itself from a puddle shape to a spherical shape before the start of the wetting phase. The width of the plateau region becomes larger as the impact velocity increases, which means the pure wetting process is delayed more at higher impact velocities. Last but not the least, the dimensionless wetting rate 
$(d \beta / d \tau)$, as indicated in Fig. 4(b) by the slope of the spreading curves in the later stage, decreases as the impact velocity increases. In the case of very low impact velocity $\left(V_{0}=\right.$ $0.2 \mathrm{~m} / \mathrm{s}$ ), the dimensionless wetting rate is similar to the droplet spreading rate.

The last discussion in this section is on the maximum spreading factor $\left(\beta_{m}\right)$. The variation of $\beta_{m}$ with respect to the Weber number $(W e)$ is demonstrated in Fig. 5(a). It is shown that the $\beta_{m}$ for Polysilazane droplet is always lower than that for water droplet at the same $W e$. Moreover, the increase of $\beta_{m}$ with increasing $W e$ appears weaker for Polysilazane droplet. The high viscosity $(\mu=32 c p)$ is believed to result in such differences in the impacting process. A universal rescaling method proposed in Refs. [9-10] is usually used for correlating the maximum spreading factor for different liquids and surfaces:

$$
\left(\beta_{m}^{2}-\beta_{V_{0} \rightarrow 0}^{2}\right)^{1 / 2} R e^{-1 / 5}=W e^{1 / 2} /\left(A+W e^{1 / 2}\right),
$$

where $A=7.6$ in this paper, which is the same as used in Ref. [10]. Figure 5(b) shows such universal scaling for the water and Polysilazane droplet on the Inconel surface. Both the data for water and Polysilazane droplets are well correlated by the scaling in Eq. (1), indicating that the impacting process of the pure liquid polymer droplet can be described well with pure hydrodynamics. Therefore, an interesting question that deserves further scrutinization is : can the subsequent wetting process also be well depicted by the classical wetting dynamics [14]?

(a)

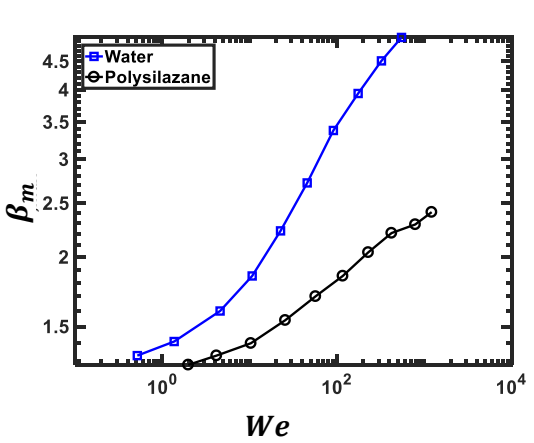

(b)

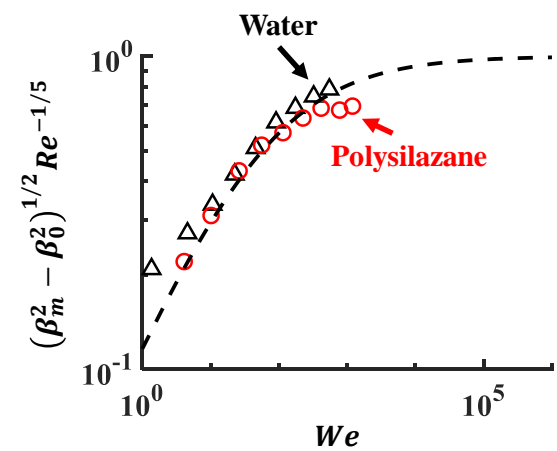

Figure 5. (a) Variation of $\beta_{m}$ with We for water and Polysilazane droplets; (b) The universal scaling correlating $\beta_{m}$ with $W e$.

After the plateau phase, the Polysilazane droplet enters the pure wetting phase. It was first demonstrated by Tanner [14] that the spreading diameter in this phase increases proportionally to $t^{1 / 10}$. Figure 4 (d) demonstrates the variation of the spreading diameter (in $\mathrm{mm}$ ) with respect to the real time (in $\mathrm{ms}$ ) after impact in a log-log scale. It shows that the slope does not remain the same for different impact velocities. A zoom-in view of the wetting phase shows that the slope is close to $1 / 10$ for low impact velocities. While for higher impact velocity $(\sim 1 \mathrm{~m} / \mathrm{s})$, the slope of the wetting rate can be lower than $1 / 10$. The dependence of the slope on impact velocities will need further study and will not be covered here. But a brief discussion on the deviation from the Tanner's law is provided as follows. First, it should be noted that the Polysilazane - Inconel system is a partial wetting system, where the advancing contact angle is significantly larger than 0 . While the Tanner's law was derived strictly for a complete wetting condition, where a macroscopic precursor film surrounds the moving contact line [14]. For partial wetting of complex liquid drops, previous studies have demonstrated more complicated relations [21-22] for the temporal evolution of drop spreading diameter rather than the simple power law with a 1/10 exponent. On the other hand, the studied Polysilazane (Durazane 1800) is moisture sensitive, because of which the crosslinking process may occur as soon as being 
in contact with the ambient air. Although it generally needs a few hours to reach the "dry-totouch" condition for a thin Polysilazane coating layer [23], it does not necessarily indicate a negligible effect of the crosslinking kinetics on the Polysilazane wetting behaviour. In the current experiment, it is shown that the post-impact wetting gets slower as the impact velocity becomes higher. And with a higher impact velocity, the Polysilazane drop tends to have a larger interface area exposed to the ambient air, which may facilitate the curing of the Polysilazane, especially at the liquid-air interface. Hence the interfacial tension of Polysilazane may have changed, which affects the subsequent wetting dynamics. In short, the hypothesis here is that the partial wetting nature and the moisture-curable property of the Polysilazane make its wetting dynamics deviate from the Tanner's law [14].

\section{Conclusions}

In this paper, the impacting and spreading dynamics of Polysilazane droplets on Inconel are, for the first time, investigated with different impact velocities. Comparison was made between the Polysilazane and water droplets with similar droplet diameter and impact velocities. Some preliminary but important conclusions can be summarized as below:

(1) The impacting and spreading process of Polysilazane droplet on the Inconel surface appear much smoother than that of water droplet, because of its high viscosity and strong adhesion characteristics on the Inconel surface. For low impact velocities, sharp decrease of contact angle exists in the early-stage impact process for water droplet because of the air layer effect. For Polysilazane droplet, its contact angle slowly decreases, and no propagation of capillary waves (disturbance) was found on its interface.

(2) In the case of high impact velocity, water droplet features strong retraction after reaching its maximum spreading position. In contrast, a plateau relaxation phase follows the spreading phase for Polysilazane droplet, and another pure wetting phase brings the Polysilazane droplet to its final equilibrium position.

(3) Some impacting and spreading characteristics of Polysilazane droplet on Inconel can be well described by the existing findings for other simple liquids. The evolution of the spreading factor in the kinematic phase obeys the classical power law $\sim t^{1 / 2}$ for both liquids. The "maximum spreading factor" by the inertial impacting can be reasonably predicted by the universal scaling law $\left(\beta_{m}^{2}-\beta_{V_{0} \rightarrow 0}^{2}\right)^{1 / 2} R e^{-1 / 5}=W e^{1 / 2} /\left(A+W e^{1 / 2}\right)$.

(4) However, questions remain for the pure wetting process of Polysilazane droplet on Inconel surface. The slope of the pure wetting curves was found to decrease with the increase of the impact velocity, which can be lower than 1/10 as used in Tanner's law. More details of the pure wetting process of such strong-adhesion liquid droplets deserve future scrutinization.

\section{References}

1. Barroso, G., Li, Q., Bordia, R. K., and Motz, G. Journal of materials chemistry A 7: 19361963 (2019).

2. Jiang, T., Hill, A., Fei, W., Wei, Y., Tellam, M., Xu, C., and An, L. Journal of the American Ceramic Society 93: 3017-3019 (2010).

3. Yu, Y., Chen, Y., Xu, C., Fang, J. and An, L. Journal of the American Ceramic Society 94: 2779-2782 (2011).

4. Clarke, D., Oechsner, M., Padture, N., Materials Research Society Bulletin, 37: 891-902 (2012).

5. Lojewski, B., Yang, W., Duan, H., Xu, C., and Deng, W. Aerosol Science and Technology 47: 146-152 (2013).

6. Rioboo, R., Marengo, M., and Tropea, C. Experiments in Fluids 33: 112-124 (2002).

7. Yarin, A. L. Annual Review of Fluid Mechanics 38: 159-192 (2006).

8. Josserand, C., and Thoroddsen, S. T. Annual Review of Fluid Mechanics 48: 365-391 (2016). 
9. Laan, N., de Bruin, K. G., Bartolo, D., Josserand, C., \& Bonn, D. Physical Review Applied 2(4): 044018 (2014).

10. Lee, J. B., Laan, N., de Bruin, K. G., Skantzaris, G., Shahidzadeh, N., Derome, D., ... and Bonn, D. Journal of Fluid Mechanics 786 (2016).

11. Wang, F., Yang, L., Wang, L., Zhu, Y., and Fang, T. Langmuir, 35: 3204-3214 (2019).

12. Wang, F., and Fang, T. Physical Review Fluids 5: 033604 (2020).

13. Wang, F., and Fang, T. Experimental Thermal and Fluid Science 98: 420-428 (2018).

14. Tanner, L. H. Journal of Physics D: Applied Physics 12: 1473 (1979).

15. Bergeron, V., Bonn, D., Martin, J. Y., and Vovelle, L. Nature 405: 772-775 (2000).

16. Huh, H. K., Jung, S., Seo, K. W., and Lee, S. J. Microfluidics and Nanofluidics 18:12211232 (2015).

17. Jung, S., Hoath, S. D., and Hutchings, I. M. Microfluidics and Nanofluidics 14: 163-169 (2013).

18. Brian, D., and Eslamian, M. Experiments in Fluids 60: 138 (2019).

19. Daerr, A., and Mogne, A. Journal of Open Research Software 4 (2016).

20. de Goede, T. C., de Bruin, K. G., Shahidzadeh, N., and Bonn, D. Physical Review Fluids 4: 053602 (2019).

21. De Ruijter, M. J., De Coninck, J., and Oshanin, G. Langmuir, 15(6), 2209-2216 (1999).

22. Lavi, B., and Marmur, A. Colloids and Surfaces A: Physicochemical and Engineering Aspects, 250(1-3), 409-414 (2004).

23. Bauer, F., Decker, U., Dierdorf, A., Ernst, H., Heller, R., Liebe, H., and Mehnert, R. Progress in organic coatings, 53(3), 183-190 (2005). 